\title{
Perception of audio-visual speech synchrony in Spanish- speaking children with and without specific language impairment
}

\author{
FERRAN PONS, \\ Departament de Psicologia Basica, Facultat de Psicologia, Universitat de Barcelona and Institute \\ for Brain, Cognition and Behaviour (IR3C), Barcelona, Spain \\ LLORENC. ANDREU, \\ Departament de Psicologia Basica, Facultat de Psicologia, Universitat de Barcelona and \\ Cognitive Neuroscience and Information Technologies Research Program, IN3, Universitat \\ Oberta de Catalunya \\ MONICA SANZ-TORRENT, \\ Departament de Psicologia Basica, Facultat de Psicologia, Universitat de Barcelona \\ LUCIA BUIL-LEGAZ, and \\ Departament de Psicologia Basica, Facultat de Psicologia, Universitat de Barcelona \\ DAVID J. LEWKOWICZ \\ Department of Psychology and Center for Complex Systems \& Brain Sciences, Florida Atlantic \\ University
}

\section{Abstract}

Speech perception involves the integration of auditory and visual articulatory information and, thus, requires the perception of temporal synchrony between this information. There is evidence that children with Specific Language Impairment (SLI) have difficulty with auditory speech perception but it is not known if this is also true for the integration of auditory and visual speech. Twenty Spanish-speaking children with SLI, twenty typically developing age-matched Spanishspeaking children, and twenty Spanish-speaking children matched for MLU-w participated in an eye-tracking study to investigate the perception of audiovisual speech synchrony. Results revealed that children with typical language development perceived an audiovisual asynchrony of $666 \mathrm{~ms}$ regardless of whether the auditory or visual speech attribute led the other one. Children with SLI only detected the $666 \mathrm{~ms}$ asynchrony when the auditory component followed the visual component. None of the groups perceived an audiovisual asynchrony of $366 \mathrm{~ms}$. These results suggest that the difficulty of speech processing by children with SLI would also involve difficulties in integrating auditory and visual aspects of speech perception.

\section{Introduction}

Whenever we interact with other people, we can usually see as well as hear them talking. As a result, everyday speech is audiovisual rather than auditory in nature. Normally, because of our ability to integrate the auditory and visual streams of speech information, we perceive them as part of a unified multisensory event (Alsius, Navarra, Campbell, \& Soto-Faraco, 2005; Lewkowicz, 2010; Munhall \& Vatikiotis-Bateson, 2004). Integration is facilitated by the fact that the dynamic auditory and visual signals that specify audiovisual speech are temporally coupled and, thus, highly redundant (Chandrasekaran, Trubanova, Stillittano, 
Caplier, \& Ghazanfar, 2009; Yehia, Rubin, \& Vatikiotis-Bateson, 1998). Once the streams of auditory and visual information are integrated, the speech becomes more salient (Sumby \& Pollack, 1954; Summerfield, 1979) and more intelligible (Munhall, Gribble, Sacco, \& Ward, 1996) and, as evidence of this, both infant (Lewkowicz \& Hansen-Tift, 2012) and adult listeners (Sumby \& Pollack, 1954; Summerfield, 1979) take advantage of the greater intelligibility of audiovisual as opposed to auditory speech.

Integration of auditory and visual speech and, by extension, its intelligibility is affected by the specific temporal relationship between the auditory and visual streams of speech information (van Wassenhove, Grant, \& Poeppel, 2007). When the auditory and visual streams of information are delayed with respect to one another, intelligibility is adversely affected in an asymmetrical fashion: when auditory speech leads visual speech intelligibility declines much more than when auditory speech follows visual speech. Importantly, however, as long as the auditory and visual components fall into what is known as the intersensory temporal contiguity window (ITCW; Lewkowicz, 1996), perceivers experience those components as part of a unitary event. The size of the ITCW differs as a function of whether the auditory component follows the visual component (V-A asynchrony) or whether it precedes it (A-V asynchrony). In adults, the ITCW is approximately 180-240 ms for a VA asynchrony but only 60-120 ms for an A-V asynchrony (Dixon \& Spitz, 1980; Munhall, Gribble, Sacco, \& Ward, 1996; van Wassenhove, Grant, \& Poeppel, 2007).

Developmental studies have found similar differences in infants although the ITCW in infancy is considerably larger (Lewkowicz, 1996, 2000, 2010). For example, when infants are habituated to a speech syllable and then tested for detection of an A-V asynchrony between its audible and visible attributes, they detect the asynchrony only when it reaches 633-666 ms. This finding indicates that the ITCW narrows with development. The developmental narrowing of the ITCW is also evident in findings from studies of responsiveness to non-speech events. Thus, infants can only detect a V-A asynchrony between a bouncing object and its impact sound when the asynchrony reaches $450 \mathrm{~ms}$ and an A-V asynchrony between the visible and audible bounce when it reaches $350 \mathrm{~ms}$ (Lewkowicz, 1996). Consistent with the developmental narrowing of the ITCW, adults can detect lower asynchronies for non-speech events (Dixon \& Spitz, 1980).

Why might the ITCW be larger for V-A than for A-V asynchrony? One reason is that whenever people speak, the motion of their lips can be seen before their vocalizations can be heard (Chandrasekaran, et al., 2009). As a result, the perceptual system expects a delay. In addition, because lip motion is of a continuous nature, it is more difficult to determine the precise point when the vocalization begins in relation to lip motion. In contrast, when a vocalization begins first, the perceptual system does not expect a delay and the punctate onset of the vocalization makes it is easier to determine that this is the point when lip motion corresponds.

If perception of the unitary nature of speech is critical for adaptive functioning and, if this depends on the ability to perceive the temporal relationship between the auditory and visual attributes of speech, then impaired detection of audio-visual temporal synchrony would be maladaptive. One specific disorder where this may be the case is specific language impairment (SLI). In general, children with SLI are characterized by developmental delays in a number of different language domains, including semantic, morphosyntactic, pragmatic, and discourse skills in oral and/or written language (Leonard, 1998). In addition, studies have found that children with SLI perform poorly on tasks requiring the processing of relatively brief $(250 \mathrm{~ms})$ synthetic CV syllables for which the critical formant transition was short in duration (40 ms) as well as stimuli presented in rapid succession (Tallal \& Piercy, $1973 ; 1975)$. Studies also have found that children with SLI have more difficulty in tasks 
requiring identification of brief stimuli than do age-matched peers (Elliott \& Hammer, 1988; Tallal, Stark, \& Mellits, 1985; Wright et al., 1997). These findings have led some researchers to argue that the deficits in processing brief sounds underlie SLI (auditory temporal processing hypothesis, Tallal, 1984). However, a number of concerns have been raised about this conclusion. For example, the tasks used by Tallal and colleagues require substantial attention and memory skills, suggesting that task specific effects might account for the Tallal et al. results (see, Elliott \& Hammer, 1988). Furthermore, some children with SLI have difficulty with particular processing tasks, but not with the processing of rapid auditory transitions (Stark \& Heinz, 1996).

Regardless of the specific auditory processing difficulties that children with SLI may have, it is likely that visual speech information might facilitate auditory processing in these children, especially when auditory perception alone is less than optimal. An examination of the empirical literature in audiovisual (AV) speech perception provides mixed clues about the ability of children with speech and language disorders to lip-read and whether they can take advantage of visual information to compensate for auditory processing difficulties. On the one hand, some studies of AV speech perception have indicated that preschool children who make developmental speech errors perform differently from their controls on lipreading tasks (Desjardins, Rogers, \& Werker, 1997). On the other hand, a study of children's response to the McGurk effect ${ }^{1}$ has found that children with speech disorders do not differ from matched controls in their perception of the illusion or in their favored strategy in response to incongruent AV speech sounds (Dodd, Mcintosh, Erdener, \& Burnham, 2008). However, it has also been reported that children with language disorders show a diminished McGurk effect relative to their peers (Boliek, Keintz, Norrix, \& Obrzut, 2010). In particular, Norrix and colleagues (Norrix, Plante, Vance, \& Boliek, 2007) found that children with SLI are less influenced by the visual information in a McGurk task than their peers and concluded that children with SLI may differ both from adults and from their normal peers in the degree to which the visual dimensions of articulated speech affect their response to audiovisual speech. If that is the case then these findings suggest that speech perception difficulties in children with SLI may not be specific to the auditory modality but may reflect an inability to respond to the combination of auditory and visual information.

Given the potential benefit of audiovisual, as opposed to auditory only, articulatory information (Sumby \& Pollack, 1954; Summerfield, 1979), it would be beneficial for children with SLI to respond to the combination of auditory and visual speech information in a manner similar to that found in their typically developing peers. One way to determine whether they do is to test their ability to perceive the temporal relationship between the audible and visible attributes of speech and to investigate whether their ITCW is similar to the ITCW in typically developing peers. Norrix et al. (2007) suggested that audiovisual integration skills in children with SLI should be investigated but, to date, no studies of this ability have appeared. As a result, in the current study we investigated the perception of audio-visual temporal synchrony in fluent speech in children with SLI and compared their performance to that of typically developing children.

Bebko, Weiss, Denmark and Gomez (2006) examined responsiveness to audiovisual temporal synchrony in speech and non-speech events in young children with autism spectrum disorder, children with other forms of developmental disability but no autism, and typically developing children. Findings indicated that typically developing children as well as children with a developmental disability but no autism preferred looking at synchronous

\footnotetext{
${ }^{1}$ The McGurk effect (McGurk \& MacDonald, 1976) is a powerful illustration that speech perception is, by default, a multisensory process where the auditory and visual information is integrated into a novel percept. For example, when a listener is presented with the sound 'ba-ba' while the lips of a speaker are silently mouthing 'ga-ga', the listener hears 'da-da'.
} 
rather than asynchronous events regardless of whether they were speech or non-speech events. In contrast, children with autism did not exhibit a preference for synchronous speech events. Critically, these children failed to detect the difference between synchrony and asynchrony even though the asynchrony was as large as three seconds, and, even though the asynchrony was far larger (i.e., $3 \mathrm{~s}$ ) than the asynchrony that infants can detect (Lewkowicz, 1996, 2000, 2010).

To determine whether children with SLI are impaired in their ability to perceive combined auditory and visual speech, we investigated their preference for one of two audiovisual speech events. One of these events showed a talker's face whose visible speech was synchronized with a concurrently presented sound track while the other event showed the same talker's face whose visible speech was desynchronized with respect to the sound track by 666 or $366 \mathrm{~ms}$. To determine whether the children with SLI are impaired, we compared their performance to typically developing children. Based on Norrix et al.'s (2007) findings, we expected that children with SLI would exhibit impaired detection of audiovisual synchrony relations compared to typically developing children.

\section{Method}

\section{Participants}

All participants were native Spanish speakers selected from state schools in Catalonia and Valencia (Spain) and did not need eye glasses to see the computer screen. Three groups took part in this study, a group of twenty children with SLI (4;04-7;02 years), a group of 20 typically developing age-matched children (4;04-6;10 years), and a group of 20 children matched for mean length of utterance (MLU-w; 3;04-6;02 years). The parents of each child gave their written informed consent prior to their child's participation in the study.

The children with SLI were diagnosed with Specific Language Impairment (SLI) by speech and language therapists from school educational psychology services and were receiving language intervention. They were selected according to standard criteria for diagnosing SLI (Leonard, 1998; Stark \& Tallal, 1981). Specifically, children with SLI were tested to assess their nonverbal intelligence and level of language development. Tests included the Wechsler Intelligence Scale for Children (WISC-R; Spanish version) (Wechsler, Cordero \& de la Cruz, 1993) or the Kaufman Brief Intelligence Test (KBIT; Spanish version) (Kaufman \& Kaufman, 1997). Every child with SLI obtained a nonverbal IQ standard score above 85. Language ability was assessed by language profiles following the Spanish protocol for evaluation of language delay, the 'Análisis del Retraso del Lenguaje' (AREL) (Pérez \& Serra, 1998), the Peabody Picture Vocabulary Test III (PPVT-III, Spanish version) (Dunn, Dunn \& Arribas, 2006) and the ELI (Early Language Inventory) child language scale (Saborit \& Julián, 2005) for children younger than 6 years. The ELI scale includes several subtests for phonetics, lexical reception, lexical production and pragmatics. Children with SLI had scores of at least a -1.25 standard deviation below the mean, both on the Peabody III and the ELI.

Language profiles based on transcripts of spontaneous conversations provided further information about the characteristics of the language production of the children. These analyses showed that these children had a delay of at least one year in language production, based on MLU-w values. Children were excluded if they had difficulty hearing pure tones in normal frequency ranges, or had neurological dysfunction, oral or motor dysfunction, or impaired social functioning. A summary of the descriptive data for the three groups of children can be found in Table 1 . 
The second group consisted of 20 children matched on age (+/ -2 months) and gender with the children with SLI. Children were not selected if they had a history of speech therapy or psychological therapy. Teachers confirmed that the control participants' language development was typical for their age. Finally, the third group consisted of 20 children matched with the children with SLI on MLU in terms of words (+/-0.6 words) and gender. In addition, nonverbal intelligence and language ability was assessed in all children selected in both the age-control and MLU-w groups using the same tests and protocols applied to children in the SLI group (seven children were not tested with the ELI scale given that they were not under 6 years). The socio-economic background of the children based on occupational status and educational degree of parents was established as a middle socioeconomic status.

\section{Apparatus}

A Tobii T120 eyetracker was used to collect and store eye-tracking data. These data consisted of the participants' eye position sampled at $120 \mathrm{~Hz}$ (approximately $8 \mathrm{~ms}$ intervals). The Tobii T120 Eye Tracker is integrated together with a 17" TFT monitor and, thus, the visual stimuli were presented on this monitor and the sound track was presented via a builtin speaker.

\section{Stimuli}

The stimuli consisted of multimedia movies which were constructed with Premiere 6.0 (Adobe Corporation) and consisted of two side-by-side video clips of the same female speaker looking directly at the camera and uttering a prepared script (see Appendix). The movies were presented at $30 \mathrm{frames} / \mathrm{sec}$ and had a resolution of $1024 \times 480$-pixels. The sound track portion of the movie was made with an audio sampling rate of $1024 \mathrm{kbps}$.

Across all the movies, one of the faces (counterbalanced for side across trials) was always synchronized with the sound track while the other one was not. In two of the movies, the sound track for the desynchronized face preceded the visual speech by $366 \mathrm{~ms}$ (A-V 366) or by $666 \mathrm{~ms}$ (A-V 666). In the other two movies, the sound track for the desynchronized face followed the visual speech by $366 \mathrm{~ms}$ (V-A 366) or by $666 \mathrm{~ms} \mathrm{(V-A} \mathrm{666).}$

\section{Procedure}

Children were tested individually at their school. They were seated approximately $22^{\prime \prime}$ in front of the Tobii T120 eyetracker. A nine point calibration was carried out at the beginning of the experiment. The Tobii Studio Software automatically validates calibrations and the experimenter could, if required, repeat the calibration process if validation was poor.

The experiment consisted of four trials during which different video clips were presented. Each trial consisted of the presentation of a synchronous clip paired with one of the following clips where the auditory and visual speech streams were desynchronized (A-V 666, A-V 366, V-A 666 and V-A 366). All clips had a duration of $30 \mathrm{~s}$. An attention-getter (a cross-hair) was presented in the middle of the screen between trials to center the children's attention prior to the next trial. Side of presentation and trial order were counterbalanced across children. Children were seated in front of the monitor and told that there were two faces talking and that one of them corresponded to the voice that they were hearing. They were given no explicit task to perform.

To acquaint the child with the procedure, a familiarization/baseline trial was presented prior to the start of the experiment-proper. The same two faces were presented during this trial with one face in synchrony and the other desynchronized with respect to the audio by one second (the audio preceded the video). All the children easily identified the synchronous 
face, indicating that they were able to solve the task. Once this baseline trial ended, the experiment began. As soon as the child fixated the attention getter, the test movies started to play.

\section{Results}

To measure preferences, we divided the screen into a left and a right area of interest (AOI) and calculated the duration of fixation that each participant directed at each AOI during each trial. To determine whether children perceived AV speech asynchrony and at what degree of asynchrony they did so, for each trial we computed the total time children spent looking at the synchronized face versus the total time they spent looking at the desynchronized face. Based on the unity assumption (Vatakis \& Spence, 2007; Welch \& Warren, 1980), according to which observers prefer concordant versus discordant multisensory events, we expected that children would be able to identify the talking face that was synchronized with the sound track and that this preference would be evident in greater looking at the synchronized face.

As indicated in the Introduction, the size of the ITCW is smaller for A-V asynchrony than for $\mathrm{V}$-A asynchrony. Consequently, we analyzed responsiveness to these two types of asynchrony separately. In the first analysis, we examined responsiveness to A-V asynchrony and, to do so, we submitted the duration of looking scores to a repeated-measures analysis of variance (ANOVA), with trial (2) and synchrony (2) as the within-subjects factors and group (3) as the between-subjects factor. The ANOVA yielded a main effect of group $[F(2,57)=$ 5.993, $\mathrm{p}=.004, \eta \mathrm{p} 2=.179$ ] which was due to less overall looking in children with SLI than in children in the two control groups. Despite the absence of any interaction involving trial as a factor, we felt that the theoretical predictions offered in the Introduction provide a strong, empirically based, a priori justification for examining the data separately for each trial (we expected that children with SLI would exhibit difficulties in detecting the specific asynchronies presented). Tests of these a priori hypotheses were conducted using Bonferroni adjusted alpha levels of .016 per test (.05/3).

Separate two-tailed t-tests revealed that the A-V_666 asynchrony was perceived by all three groups; the children looked longer at the synchronized than at the desynchronized face [Age-Control: t $(15)=2.669, \mathrm{p}=.002$; MLU-w Control: $\mathrm{t}(16)=3.118, \mathrm{p}=.007$; SLI: t (19) $=2.402, \mathrm{p}=.003]$. Looking times of each group are shown in Table 2 and illustrated in Figure 1. For the A-V 366 trial, two-tailed t-tests indicated that, given the Bonferroni adjustment, the A-V 366 asynchrony was not detected by any of the groups [Age-Control: $t$ $(19)=.963, p=0.347 ;$ MLU-w Control: $t(19)=3.338, p=.02$, Cohen's $d=0.60$, medium effect size; SLI: $\mathrm{t}(18)=1.255, \mathrm{p}=.225]$.

The same repeated-measures analysis of variance (ANOVA) on the data from the V-A asynchrony trials also revealed only a main effect of group $[\mathrm{F}(2,57)=4.219, \mathrm{p}=.02, \eta \mathrm{p} 2=$. 129]. As before, despite the absence of a significant interaction, we used t-tests to explore our a priori hypothesis (alpha level of .016). The analyses of the V-A 666 trial indicated that this degree of asynchrony was detected by the two control groups but not by the SLI group [Age-Control: t $(19)=2.594, \mathrm{p}=.008$; MLU-w Control: $\mathrm{t}(19)=3.216, \mathrm{p}=.01$; SLI: $\mathrm{t}(19)=$ $1.623, \mathrm{p}=.121]$. The t-tests also indicated that none of the groups detected the V-A 366 asynchrony [Age-Control: $\mathrm{t}(16)=1.594, \mathrm{p}=.130$; MLU-Control: $\mathrm{t}(16)=1.482, \mathrm{p}=.158$; SLI: $\mathrm{t}(19)=1.177, \mathrm{p}=.254]$.

The three groups detected an asynchrony of $666 \mathrm{~ms}$ when the voice led lip motion. Furthermore, only the two control groups detected an asynchrony of $666 \mathrm{~ms}$ when lip 
motion led the voice. Finally, none of the groups were able to detect an asynchrony of 366 ms.

\section{Discussion}

The purpose of the current study was to investigate the detection of audiovisual fluent speech asynchrony in children with and without SLI. To do so, children watched side-byside faces of the same person mouthing a short and identical passage and heard the person's talking at the same time. The person's vocalizations were synchronized with one of the two faces and desynchronized with the other face either by 366 or $666 \mathrm{~ms}$. As predicted, we found that children with SLI exhibited poorer detection of audiovisual asynchrony than did children without SLI. That is, whereas the children in the two control groups preferred the synchronized face and voice in the $666 \mathrm{~A}-\mathrm{V}$ and V-A asynchrony conditions, children with SLI only preferred the synchronized face and voice in the $666 \mathrm{~ms}$ A-V asynchrony condition. None of the children preferred the synchronized face and voice in the A-V and VA $366 \mathrm{~ms}$ condition, indicating that none of them detected an asynchrony of $366 \mathrm{~ms}$ regardless of whether the auditory or visual speech attribute led the other one.

The fact that children with SLI could not detect the difference between two identical talking faces based on whether the concurrent voice they were hearing corresponded to one of them or not indicates impaired perception of audiovisual temporal relations. This impairment may be due to difficulties in auditory processing, an inability to speech-read, and/or attentional control problems. With specific regard to speech perception difficulties, the present results suggest that, at a minimum, the typical kinds of speech perception difficulties that children with SLI have may not be solely due to problems in auditory processing. In other words, in addition to difficulties in auditory processing (Tallal, 1984) there may be other factors that may be responsible for speech perception difficulties in children with SLI.

The current findings suggest that children with SLI are not only impaired in their speech perception abilities but also in their processing of the temporal coherence of auditory and visual speech information. One possible reason for this may be because these children are poorer at speech-reading. Recent studies of selective attention to audiovisual speech in infancy have shown that at about six months of age when infants babble, they shift their attention to the lips of their interlocutors and continue to focus on the speaker's lips until nearly 12 months of age (Lewkowicz \& Hansen-Tift, 2012). The correlation between the sustained period of focusing on the speaker s lips between six and twelve months of age and the development of speech production capacity during that time surely contributes in important ways to the development multisensory perception. That is, they increase the opportunity to experience the temporal coherence of audiovisual speech. This fact raises the possibility that children with SLI may be impaired in the detection of audiovisual synchrony because they may not have attended to the mouth of their interlocutors during infancy as much as children without SLI. This, in turn, may make it difficult for children with SLI to integrate the articulatory code associated with visual and heard speech (Desjardins et al., 1997; Siva et al., 1995). Further studies should explore this possibility.

Finally, it may be that the attentional control difficulties of children with SLI (Noterdaeme et al., 2001) make it difficult for them to detect audiovisual synchrony relations. That is, although children with SLI are able to attend to the stimuli, they may not be able to divide their attention between simultaneously occurring congruent and incongruent visual and auditory stimuli as efficiently as typically developing children do. It should be noted, however, that this explanation does not account for the fact that these children can detect the A-V 666 asynchrony. Thus, although attentional problems may have contributed in some subtle way to the deficit, they do not appear to play a major role in it. 
In sum, the current study has found that children with SLI are poorer than their typically developing peers in their ability to distinguish between temporally coherent and incoherent auditory and visual attributes of speech. The reasons for this impairment are currently not clear; further research is required.

\section{Acknowledgments}

This work was supported by the Spanish Ministerio de Ciencia e Innovación (SEJ2007-62743 to M.S, and PSI2010-20294 to F.P) and by the National Science Foundation (grants BCS0751888 to D.J.L.). Special thanks to all the children who participated and to CEIP Els Pins (Barcelona), CREDA Narcís Massó (Girona), and the School of Educational Psychology Services -SPE (Castelló).

\section{References}

Alsius A, Navarra J, Campbell R, Soto-Faraco S. AV integration of speech falters under high attention demands. Current Biology. 2005; 15:839-843. [PubMed: 15886102]

Bahrick LE, Lickliter R, Flom R. Intersensory Redundancy Guides the Development of Selective Attention, Perception, and Cognition in Infancy. Current Directions in Psychological Science. 2004; 13(3):99-102.

Bebko JM, Weiss JA, Denmark JL, Gomez P. Discrimination of temporal synchrony in intermodal events by children with autism and children with developmental disabilities without autism. Journal of Child Psychology and Psychiatry. 2006; 47(1):88-98. [PubMed: 16405645]

Boliek C, Keintz CK, Norrix LW, Obrzut J. Auditory-visual perception of speech in children with learning disabilities: The McGurk Effect. Canadian Journal of Speech-Language Pathology and Audiology. 2010; 34(6):124-131.

Chandrasekaran C, Trubanova A, Stillittano S, Caplier A, Ghazanfar AA. The natural statistics of audiovisual speech. PLoS Computational Biology. 2009; 5:e1000436. [PubMed: 19609344]

Desjardins R, Rogers J, Werker JF. An exploration of why Preschoolers perform differently than do adults in AV perception tasks. Journal of Experimental Child Psychology. 1997; 66:85-110. [PubMed: 9226935]

Dixon NF, Spitz LT. The detection of auditory visual desynchrony. Perception. 1980; 9:719-721. [PubMed: 7220244]

Dodd B, McIntosh B, Erdener D, Burnham D. Perception of the auditory-visual illusion in speech perception by children with phonological disorders. Clinical Phonetics \& Linguistics. 2008; 22:6982.

Dunn, LlM; Dunn, LM.; Arribas, D. PPVT-III. Peabody. Test de vocabulario en imágenes. Madrid: TEA Ediciones; 2006.

Elliott LL, Hammer MA. Longitudinal changes in auditory discrimination in normal children and children with language-learning problems. Journal of Speech and Hearing Disorders. 1988; 53:467-474. [PubMed: 3263544]

Kaufman, AS.; Kaufman, NL. KBIT: Kaufman Brief Intelligence Test (KBIT, Spanish version). Madrid: TEA Editions; 2004.

Lewkowicz DJ. Perception of auditory-visual temporal synchrony in human infants. Journal of Experimental Psychology: Human Perception \& Performance. 1996; 22(5):1094-1106. [PubMed: 8865617]

Lewkowicz DJ. The development of intersensory temporal perception: An epigenetic systems/ limitations view. Psychological Bulletin. 2000; 126(2):281-308. [PubMed: 10748644]

Lewkowicz DJ. Infant perception of audio-visual speech synchrony. Developmental Psychology. 2010; 46 (1):66-77. [PubMed: 20053007]

Lewkowicz DJ, Hansen-Tift AM. Infants deploy selective attention to the mouth of a talking face when learning speech. Proceedings of the National Academy of Sciences. 2012; 109(5):14311436. 
Lewkowicz, DJ.; Kraebel, K. The value of multimodal redundancy in the development of intersensory perception. In: Calvert, G.; Spence, C.; Stein, B., editors. Handbook of multisensory processing. Cambridge: MIT Press; 2004.

McGurk H, MacDonald J. Hearing lips and seeing voices. Nature. 1976; 264:246-748.

Munhall K, Gribble P, Sacco L, Ward M. Temporal constraints on the McGurk effect. Perception and Psychophysics. 1996; 58:351-362. [PubMed: 8935896]

Munhall, KG.; Vatikiotis-Bateson, E. Spatial and Temporal Constraints on Audiovisual Speech Perception. In: Calvert, GA.; Spence, C.; Stein, BE., editors. The handbook of multisensory processes. Cambridge, MA: MIT Press; 2004. p. 177-188.

Norrix LW, Plante E, Vance R, Boliek CA. Auditory-visual integration for speech by children with and without specific language impairment. Journal of Speech, Language and Hearing Research. 2007; 50:1639-1651.

Noterdaeme M, Amorosa H, Mildenberger K, Sitter S, Minow F. Evaluation of attention problems in children with autism and in children with specific language disorder. European Child and Adolescent Psychiatry. 2001; 10:58-66. [PubMed: 11315537]

Osterling JA, Dawson G, Munson JA. Early recognition of 1-year-old infants with autism spectrum disorder versus mental retardation. Development and Psychopathology. 2002; 14(2):239-251. [PubMed: 12030690]

Partan S, Marler P. Communication goes multimodal. Science. 1999; 283(5406):1272-1273. [PubMed: 10084931]

Perez, E.; Serra, M. Análisis del retraso del lenguaje (AREL). Barcelona: Ariel; 1998.

Saborit, C.; Julin, JP. ELI - La evaluación del lenguaje infantil. Castello de la Plana: Universitat Jaume I; 2003.

Siva N, Stevens E, Kuhl P, Meltzoff A. A comparison between cerebralpalsied and normal adults in the perception of auditory-visual illusions. Journal of the Acoustical Society of America. 1995; 98:2983.

Stark RE, Tallal P. Selection of children with specific language deficits. Journal of Speech and Hearing Disorders. 1981; 46:114-122. [PubMed: 7253588]

Stein BE, Stanford TR. Multisensory integration: current issues from the perspective of the single neuron. Nature Review Neuroscience. 2008; 9(4):255-266.

Tallal P. Temporal or phonetic processing deficit in dyslexia? That is the question. Applied Psycholinguistics. 1984; 5:167-169.

Tallal P, Piercy M. Developmental aphasia: Impaired rate of non-verbal processing as a function of sensory modality. Neuropsychologia. 1973; 11:389-398. [PubMed: 4758181]

Tallal P, Piercy M. Developmental aphasia: The perception of brief vowels and extended stop consonants. Neuropsychologia. 1975; 13:69-74. [PubMed: 1109463]

Tallal P, Stark R, Mellits E. Identification of language-impaired children on the basis of rapid perception and production skills. Brain and Language. 1985; 25:314-322. [PubMed: 2415209]

van Wassenhove V, Grant KW, Poeppel D. Temporal window of integration in auditory-visual speech perception. Neuropsychologia. 2007; 45(3):598-607. [PubMed: 16530232]

Vatakis A, Spence C. Crossmodal binding: Evaluating the "unity assumption" using audiovisual speech stimuli. Perception \& Psychophysics. 2007; 69:744-756. [PubMed: 17929697]

Wechsler, D.; Cordero, A.; de la Cruz, MV. WISC-R: escala de inteligencia de Wechsler para niños revisada: manual. Madrid: TEA Ediciones; 1993.

Welch RB, Warren DH. Immediate perceptual response to intersensory discrepancy. Psychological Bulletin. 1980; 88:638-667. [PubMed: 7003641]

Yehia H, Rubin P, Vatikiotis-Bateson E. Quantitative Association of Vocal-Tract and Facial Behavior. Speech Communication. 1998; 26(1-2):23-43.

\section{Appendix}

Script: ;Buenos días, despiértate ya! ¡Si te levantas ahora tendremos una hora entera para jugar! Me encantan estas mañanas largas, ¿y a ti? Ojala no se acabaran nunca. Bueno, por 
lo menos es viernes y tenemos todo el sábado para descansar, excepto por lo de la fiesta. Me vas a ayudar a arreglar la casa, ¿si? Tenemos que comprar flores, preparar la comida, sacar el polvo, aspirar la casa y limpiar los discos'.

English translation: Good morning! Get up. Come on now. If you get up right away, we have a whole hour to putter around the house. I love these long mornings, don't you? I wish that they could last all day. Well, at least it's Friday and we can loaf around all day Saturday, except of course, for the party. Are you going to help me fix up the house? We have to buy flowers, prepare the food, vacuum the house, dust everything and clean the records). 
AV_666

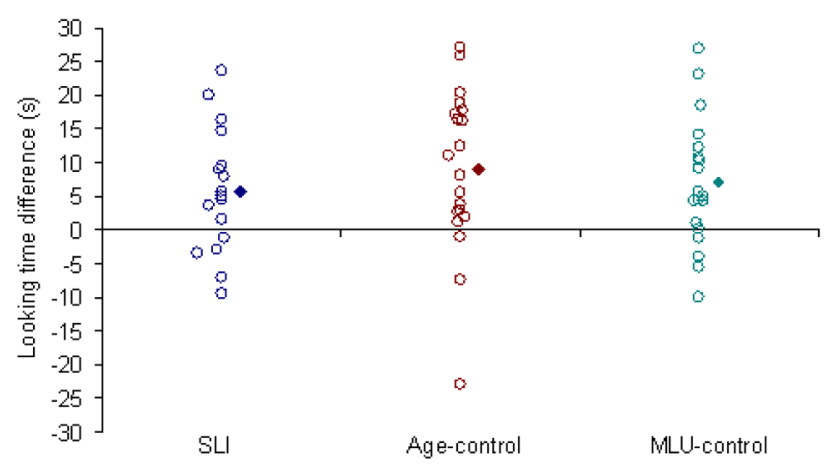

Av_366

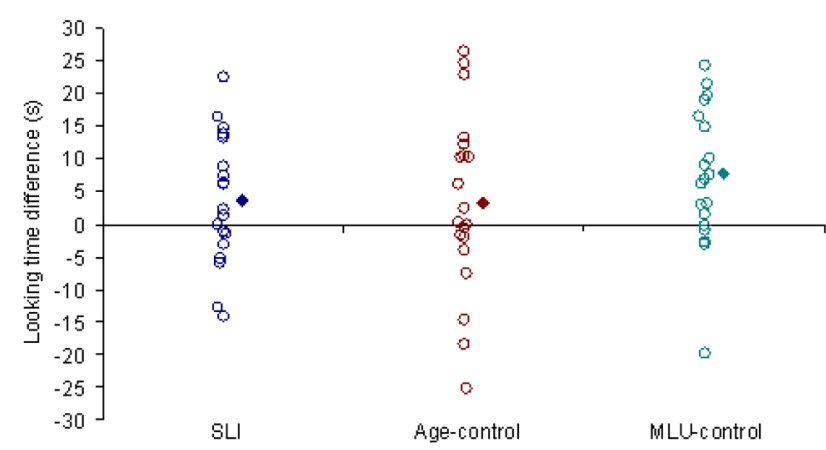

Av_-666

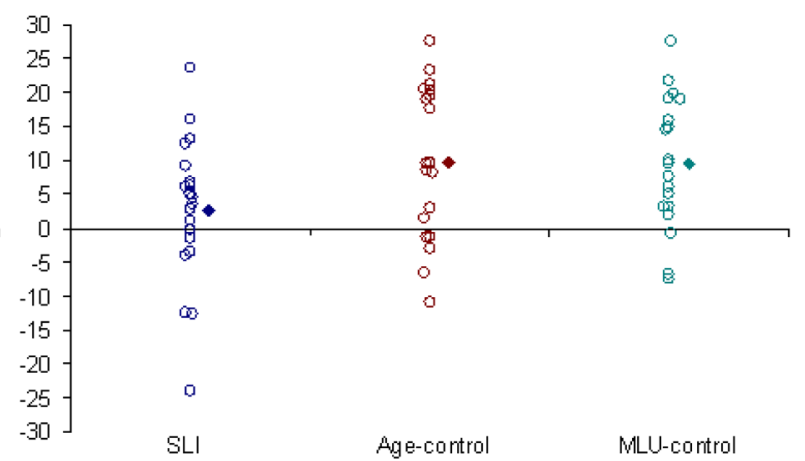

AV_-366

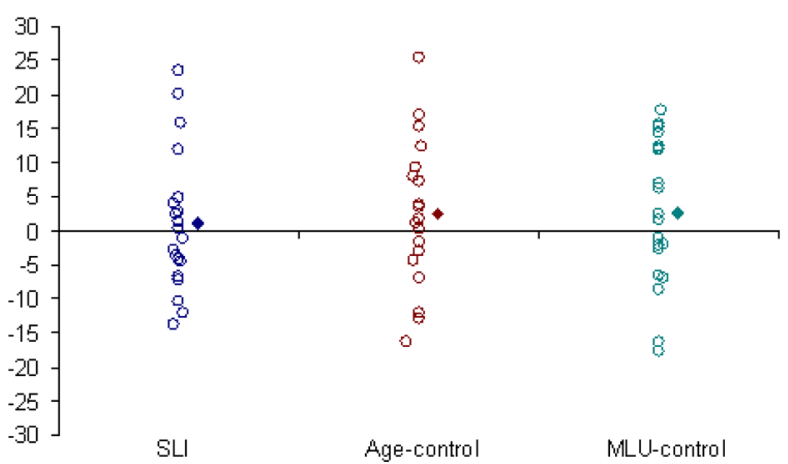

Figure 1.

Distribution of looking time difference scores (in seconds) to the synchronized face for each type of trial and children group. Open circles represent each child's score. Filled diamonds represent mean difference score for each group. 
Table 1

Group age, cognitive measures and language performance.

\begin{tabular}{cccc}
\cline { 2 - 4 } & SLI group & Age control & MLUw Control \\
\cline { 2 - 4 } & Mean (S.D.) & Mean (S.D.) & Mean (S.D.) \\
Age (years) & $6.69(0.90)$ & $6.72(0.92)$ & $5.51(1.05)$ \\
NVIQ & $96.1(7.9)$ & $106.3(6.0)$ & $93.13(9.32)$ \\
PPVT-III & $77.45(8.96)$ & $112.07(14.37)$ & $92(12.87)$ \\
ELI-Receptive vocabulary $^{*}$ & $36.27(18.84)$ & $73.07(17.97)$ & $67.85(26.13)$ \\
ELI-Expressive vocabulary $^{*}$ & $8.62(1.8)$ & $60.38(15.06)$ & $52.27(28.84)$ \\
MLUw & $3.95(1.39)$ & $6.86(1.76)$ & $3.46(1.55)$ \\
\hline
\end{tabular}

Note. Chronological age in years; NVIQ (Nonverbal Intelligence Quotient) in standard score (mean=15; SD: 15); PPVT-III (Peabody Picture Vocabulary Test III. Spanish version) in standard score (mean=15; SD: 15); ELI (Evaluación del Lenguaje Infantil); ELI-Phonetics in mean number of errors; ELI-Receptive vocabulary. ELI-Expressive vocabulary and ELI-Pragmatics in percentiles; MLU-w (Mean Length of Utterance by words).

* Values only calculated for children younger than 6 years old. 


\section{Table 2}

Mean and Standard Deviations of the looking time children spent looking at the synchronized face versus the total time they spent looking at the desynchronized face.

\begin{tabular}{|c|c|c|c|c|}
\hline & \multicolumn{2}{|c|}{ A-V design } & \multicolumn{2}{|c|}{ V-A design } \\
\hline & $A-V 666$ & $A-V 366$ & $V-A 666$ & $V-A 366$ \\
\hline Children with SLI & $16.3(6.2) / 10.2(5.8)$ & $11.1(6.7) / 12.3(7.3)$ & $13.4(7.0) / 11.7(6.8)$ & $14.0(6.1) / 13.2(6.1)$ \\
\hline Age-Control & $19.1(5.8) / 11.7(6.0)$ & $15.3(6.5) / 14.6(6.8)$ & $17.3(5.4) / 12.5(5.1)$ & $14.3(7.3) / 15.5(5.9)$ \\
\hline MLU-w Control & $18.3(6.1) / 11.3(5.7)$ & $15.1(4.9) / 11.0(5.6)$ & $18.6(4.9) / 13.3(5.9)$ & $14.3(6.3) / 14.1(6.8)$ \\
\hline
\end{tabular}

Bold numbers indicate significant effects.

$* \leq .01$. 